\title{
Regional ventilation distribution in the first 6 months of life
}

\author{
T.M.T. Pham*, M. Yuill\#, C. Dakin" and A. Schibler*
}

ABSTRACT: Electrical impedance tomography (EIT) has been used to study regional ventilation distribution in neonatal and paediatric lung disease; however, little information has been obtained in healthy newborns and infants.

Data on regional ventilation distribution and regional filling characteristics were obtained using EIT in the neonatal period, at 3 and 6 months of age, in spontaneously breathing infants during non-rapid eye movement sleep. Regional ventilation distribution was described using regional end-expiratory and end-inspiratory impedance amplitudes, and geometric centre of ventilation. Regional filling characteristics were described with the phase lag or lead of the regional impedance change in comparison to global impedance change.

32 infants were measured in the supine position. Regional impedance amplitudes increased with age but regional ventilation distribution remained unchanged in all infants at any age, with the dependent (posterior) lung always better ventilated. Regional filling characteristics showed that the dependent lung filled during inspiration before the nondependent lung during all followup measurements.

Regional ventilation distribution and regional filling characteristics remained unchanged over the first 6 months of life, and the results obtained on regional ventilation distribution are very similar to those in adult subjects.

KEYWORDS: Electrical impedance tomography, healthy reference, infant, respiratory mechanics, sigh, ventilation distribution

\footnotetext{
A better understanding of normality is needed for deriving thresholds of clinical significance for disease [1]. The rapid lung growth occurring over the first few years of life necessitates repeated measurements to understand the developmental changes of lung growth and physiological behaviour [2]. The performance of such measurements has been technically difficult, due to the inability of infants and young children to cooperate with testing and the lack of repeatable noninvasive tests [3]. Although there is a sound body of literature describing the respiratory mechanics of neonates and infants in disease [3-5], much knowledge is yet to be obtained in spontaneously breathing healthy infants. In this age group, the traditional methods of measuring respiratory mechanics, in particular regional ventilation distribution, pose some difficulties [6-8]. Multiple-breath inert gas washout techniques are commonly used to assess ventilation distribution, but cannot provide information about regional ventilation distribution [5, 8]. Other methods, such as computed tomography
}

(CT) and aerosol dispersion, involve exposure to radiation $[9,10]$.

Electrical impedance tomography (EIT) has emerged as a noninvasive functional imaging modality that can be used to measure regional ventilation distribution [11]. EIT can be used repeatedly for extended periods of patient monitoring, without the need for sedation. One of the distinct advantages of EIT measurements is that data can be analysed over a given time period and reported as an EIT image, similarly to a CT scan, or analysed on a breath-by-breath basis, to analyse the time course of regional filling $[12,13]$. This aspect is important for lung function measurements in infants, where irregular breathing and sighs are a common finding [1]. This paper reports the results of follow-up EIT measurements from a small cohort of healthy newborns at 2 weeks, and at 3 and 6 months of life measured in non-rapid eye movement (NREM) sleep. The questions that have been addressed are: 1) does regional ventilation distribution change within the first 6 months of life; 2) does regional filling

\section{AFFILIATIONS}

*Paediatric Critical Care Research Group, Paediatric Intensive Care Unit, and

\#Dept of Respiratory and Sleep Medicine, Mater Children's Hospital Brisbane, Australia.

CORRESPONDENCE

A. Schibler

Paediatric Intensive Care Unit Mater Children's Hospital South Brisbane 4101 QLD Australia E-mail: andreas.schibler@ mater.org.au

Received: March 042010 Accepted after revision: June 292010 First published online: July 222010 
change; and 3) what is the impact of a sigh on regional ventilation distribution?

\section{MATERIAL AND METHODS \\ Study design}

This study was undertaken as part of a larger prospective healthy reference cohort study from birth to 2 yrs of age, in which healthy term neonates were enrolled following a normal delivery. Regional ventilation distribution with EIT was evaluated during regular quiet breathing in NREM sleep at 2 weeks, and 3 and 6 months of age.

\section{Subjects}

EIT measurements were taken in the supine position at 2 weeks, and 3 and 6 months of age in 32 term infants (17 males and 15 females) in the sleep laboratory of the Mater Children's Hospital, Brisbane, Australia. Inclusion criteria were nonsmoking families without a history of allergies or pulmonary disease and without pre- or perinatal maternal medical history. The study had the approval of the local research ethics committee and written informed consent was obtained. A polysomnography and sleep staging was performed according to recommendations [14].

\section{EIT}

A Goe-MF II system (Cardinal Health, Hoechberg, Germany) was used to perform EIT measurements in the supine position for durations of $10 \mathrm{~min}$. The principles of EIT systems have been published elsewhere [11, 12, 15, 16]. All EIT measurements were taken with electrodes at nipple level and were sampled at 13 images $\cdot \mathrm{s}^{-1}(13 \mathrm{~Hz})$ with a $50 \mathrm{kHz}$ injection current of $5 \mathrm{~mA}$ peak-to-peak. EIT data were referenced to a regular tidal breathing period during quiet NREM sleep. Data were analysed offline using a custom-developed EIT Data Analyser program based in MATLAB v7.7 (The Mathworks Inc., Natick, MA, USA).

\section{Image analysis}

EIT data were band-pass filtered, inclusive of the first and second harmonics of the respiratory rate $[12,17,18]$, and a cutoff mask at $20 \%$ of the peak impedance signal applied [19]. EIT images were generated using the average end-expiratory to end-inspiratory impedance differences for each individual pixel time-course array to describe the magnitude of the regional tidal volume change among individuals. Six slices from anterior to posterior (A-P axis) and from right to left ( $\mathrm{R}-\mathrm{L}$ axis) were defined as regions of interest (ROI)1-6 for regional impedance amplitude analysis [15]. EIT images and regional amplitudes were obtained for $\geqslant 10$ consecutive breaths during quiet breathing. Additionally, the regional impedance amplitudes were measured prior to, during and after a sigh in NREM sleep. The geometric centre of the EIT image was calculated for the entire image, and for the right and left lung separately [20]. The geometric centre, based on the $32 \times 32$ matrix, defines the centre of ventilation using a balanced averaging of pixel values from anterior to posterior or from right to left.

\section{Time-course analysis}

Regional filling characteristics of the lung can be measured using the time-course of the impedance measurements. An ROI impedance change may show a phase lead or lag in relation to the whole lung. Such phase shifts were described with phase angles [21]. If an ROI fills ahead of the rest of the lung, the phase angle is positive. If an ROI fills after the rest of the lung, the phase angle becomes negative. We used a cross-correlation method to calculate the phase angles $[12,22]$.

\section{Analysis of sighs}

The regional impedance amplitudes were calculated for 10 breaths prior to and after a sigh, and also during the sigh itself. The regional volume expansions of the tidal breaths and the sigh were compared to global volume expansion by plotting the impedance change of the anterior or posterior lung against that of the global signal, forming a curve [13]. A linear relationship is found if the percentile degree of volume change of an ROI is the same as that of the global lung. If the rate of change in an ROI is initially less but then, towards the end of the inspiratory effort, greater than the global lung, then the curve has a concave shape. If the rate of expansion in a ROI during the initial phase of the inspiration is greater than the global lung but decreases towards the end, then the curve has a convex shape. In order to quantify this physiological behaviour, a curve fit was performed [23] using the formula:

$$
I(g)=a g^{\mathrm{FI}}+c
$$

where $I(g)$ is the impedance change of the ROI, $g$ is the impedance change of the global signal, FI is the filling index of the ROI, and $a$ and $c$ are constants. FI describes the shape of the curve, where a convex curvature is evident when FI $<1$ and a concave shape is evident when FI $>1$; thus, physiological behaviour observed. To analyse the impact of a sigh on regional filling characteristics, the FIs of the anterior and posterior lung were calculated for 10 breaths prior to, during and 10 breaths after a sigh.

\section{Statistics}

Data are presented as mean (95\% CI). A one-way ANOVA with a Bonferroni correction was applied for repeated measurements.

A multi-way ANOVA was performed to assess the effect of sex, age and region of interest on regional amplitudes, geometric centre and phase angles. A paired t-test was used for comparison of variables within different lung regions. For statistical analysis, SPSS version 15.0 (SPSS Inc., Chicago, IL, USA) was used.

\section{RESULTS}

\section{Feasibility of measurements}

All 32 enrolled infants were assessed with EIT during NREM sleep at mean \pm SD $13.7 \pm 3$ days; 25 infants were followed up at 3 months (age $97 \pm 8$ days) and 26 infants at 6 months (age $187 \pm 6$ days). In each infant, at least one 10-min EIT measurement was obtained at each recording session.

\section{Image analysis}

Overall, there were significant increases in regional amplitudes with age for all ROIs investigated in the A-P and $\mathrm{R}-\mathrm{L}$ axes $(\mathrm{p}<0.001$ by multi-way ANOVA) (fig. 1). Inspection of regional differences showed that the measured ROI amplitudes in the gravity (A-P) axis increased, with the greatest change between 2 weeks and 3 months of age for all measured ROIs $(\mathrm{p}<0.05$ by one-way ANOVA with Bonferroni correction), but then only a 

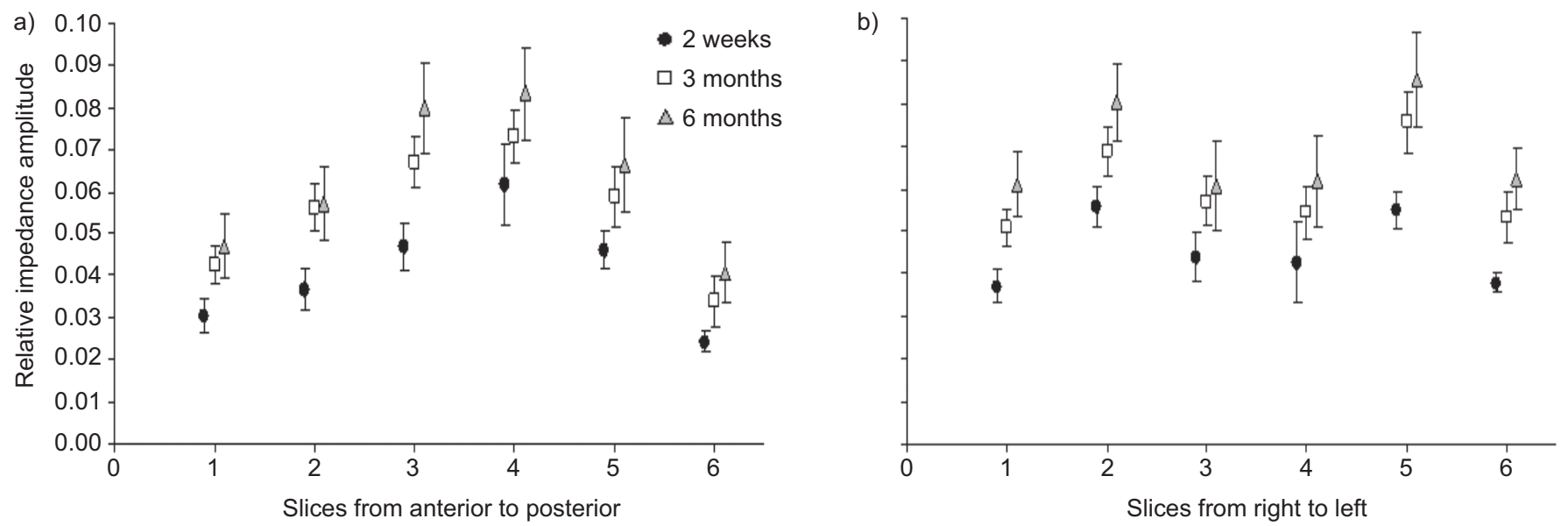

FIGURE 1. Regional impedance amplitudes increased overall between 2 weeks and 6 months of age $(p<0.001)$, with the greatest increase in regional amplitudes between 2 weeks and 3 months of age. a) Anterior-to-posterior axis. b) Right-to-left axis. Data are presented as mean $(95 \% \mathrm{Cl})$.

moderate increase was found between the ages of 3 and 6 months (not significant). The greatest regional amplitude change with growth was found in the posterior (dependent) lung, with an mean amplitude of 0.045 (95\% CI 0.006$)$ at 2 weeks, $0.055(95 \% \mathrm{CI} 0.006)$ at 3 months and $0.066(95 \% \mathrm{CI}$ $0.008)$ at 6 months of age $(\mathrm{p}<0.001)$. In the anterior (nondependent) lung, the mean amplitude increased similarly but to a lesser extent, from 0.039 (95\% CI 0.004$)$ at 2 weeks to 0.054 (95\% CI 0.004$)$ at 3 months and to 0.060 (95\% CI 0.007) at 6 months of age. In the $\mathrm{R}-\mathrm{L}$ axis, the regional amplitudes increased with age similarly to the A-P axis, with the greatest change between 2 weeks and 3 months, but a lesser change between 3 and 6 months of age. The geometric centre remained, over the entire age range, in the centre of the chest with a slight trend in location toward the anterior lung (fig. 2).

\section{Time-course analysis}

The phase angles for the anterior (nondependent) lung were predominately negative and those for the posterior (dependent) lung were positive, indicating that, during spontaneous breathing, the dependent lung filled before the nondependent lung ( $p<0.05)$ (fig. 3). A similar filling pattern was found at 3 and 6 months of age $(p<0.05)$. The comparison of the filling pattern between right and left showed that the right lung filled before the left lung in all age groups $(p<0.05)$.

\section{Analysis of sighs}

For each infant, at least two sighs were considered for analysis at each age. The measured regional impedance amplitudes of tidal breaths before and after a sigh were not different (not significant) for all age groups (fig. 4). The regional impedance amplitude of the sigh was significantly greater than that of the tidal breaths, but showed a similar regional distribution to the posterior lung showing the greatest impedance amplitudes. The FIs of the posterior and anterior lung showed a very characteristic behaviour in all investigated infants prior to and after a sigh. The FIs of the posterior lung were significantly less than 1.0, indicating that the rate of volume change in the dependent lung is greater at the beginning of the inspiration compared with the rest of the lung. The FIs of the anterior lung before and after the sigh were greater than 1.0, showing a reduced initial rate of volume change in the nondependent lung. The FI of the sigh itself showed the opposite value to the tidal breaths before and after the sigh. This pattern was similar for all age groups (table 1).

\section{DISCUSSION}

EIT has emerged as a new noninvasive lung function monitoring tool, but its role in assessing the progression of lung disease remains unclear and is a topic of many recent studies [11]. Most published studies present data obtained in mechanically ventilated subjects or patients with lung disease. Few reports document EIT measurements in healthy subjects and none yet exist for follow up studies. We investigated healthy newborn infants and followed them up with EIT measurements to provide normal data.

\section{EIT}

Image analysis

The investigation of the change in regional ventilation over the first 6 months of age along the gravity (A-P) axis showed that the posterior (dependent) lung was slightly better ventilated

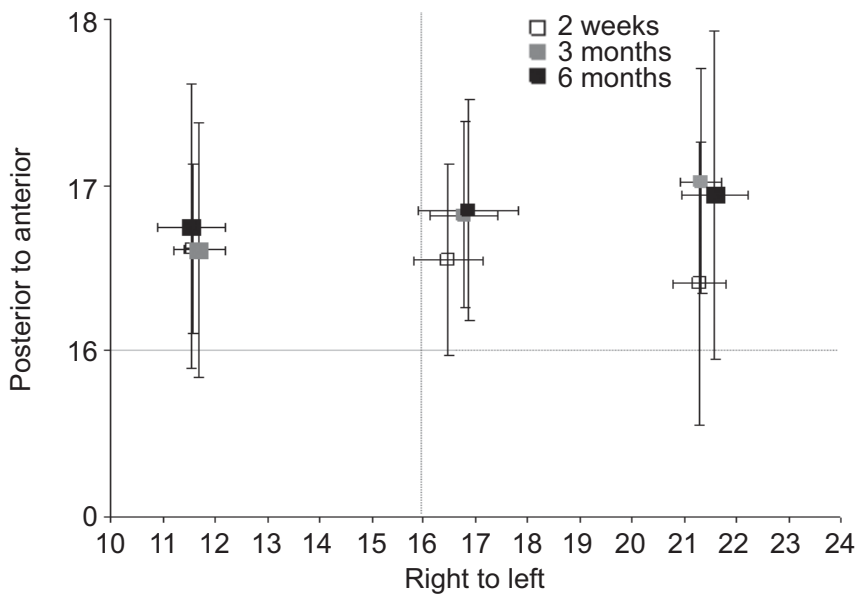

FIGURE 2. Global, and left- and right-lung geometric centres. The geometric centre remained centrally located at 2 weeks, and 3 and 6 months of age. Data are presented as mean $(95 \% \mathrm{Cl})$. 


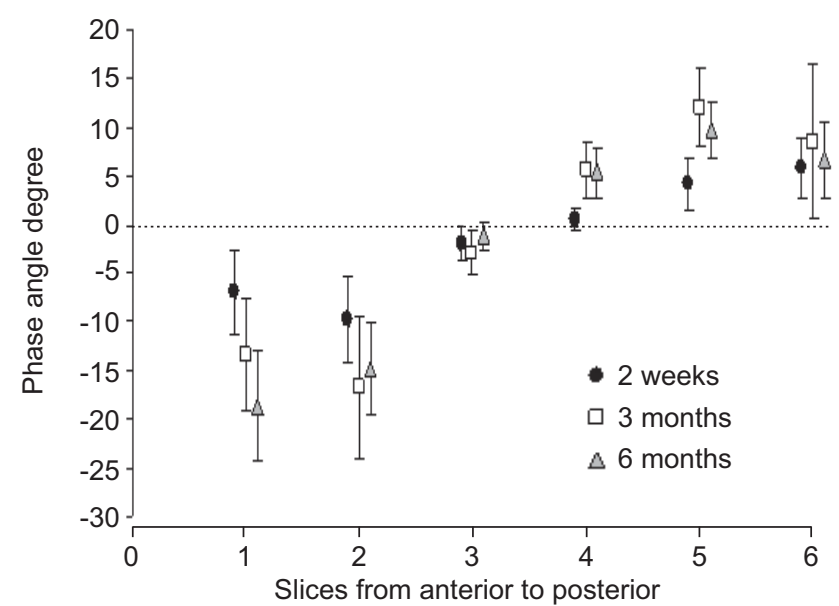

FIGURE 3. Regional filling characteristics described by phase angles. A positive value indicates that a region of interest (ROI) fills ahead of the rest of the lung, while a negative value indicates that an $\mathrm{ROI}$ fills after the rest of the lung. Data are presented as mean $(95 \% \mathrm{Cl})$.

than the anterior (non-dependent) lung, irrespective of age. Figure 1 shows that the greatest increase in ventilation between 2 weeks and 3 months of age occurred in the posterior lung. In the $\mathrm{R}-\mathrm{L}$ axis, a similar increase in regional ventilation could be observed, with both lungs contributing a similar amount. Two aspects of these findings need to be discussed. First, age-specific normal values for the magnitude of regional ventilation were found, with the dependent lung preferentially better ventilated in all age groups. BROWN et al. [24] described similar maturational changes in absolute lung resistivity with serial EIT measurements and showed that resistivity increases with age. Secondly, there is a proportionally larger increase in the magnitude of regional ventilation in the posterior (dependent) than in the anterior (nondependent) lung with growth. These findings contradict conventional wisdom on neonatal ventilation distribution, which states that the nondependent lung is preferentially ventilated. HEAF et al. [25] investigated a small cohort of infants and children with lung disease using a radiolabelled tracer gas (krypton-81m) method, and found that infants and children had the reverse ventilation pattern to adults [25]. FRERICHS et al. [15] were the first to challenge these findings and were able to demonstrate, with EIT measurements, that ventilation in infants is more centrally located. We have previously described a similar ventilation distribution in a small cohort of newborn infants [18]. EIT and krypton-81m ventilation

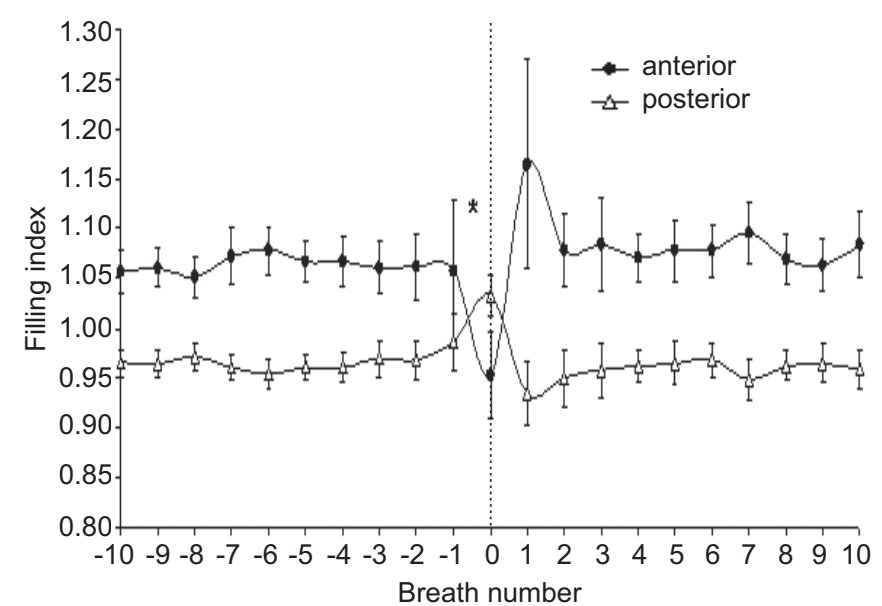

FIGURE 4. Filling indices averaged for all newborns (2 weeks of age) calculated for the anterior and posterior lung for the 10 breaths prior to the sigh the sigh iself and 10 breaths after the sigh. Data are presented as mean $(95 \% \mathrm{Cl})$. *: $p<0.05$ for the sigh itself compared with before and after.

scanning have some distinct differences in the way they measure lung volume and regional ventilation. In healthy subjects, the alveolar lung volume remains almost constant during tidal breathing and most of the volume changes (convection) during tidal breathing occur in the central and peripheral airways, and alveolar ducts [26]. EIT measurements are based on tidal volume change. Since the alveolar volume in healthy subjects is hardly changing, EIT can not identify these regions unless alveolar recruitment occurs during tidal breathing. Krypton- $81 \mathrm{~m}$ ventilation scanning investigates steady-state ventilation images based on the inhalation of the tracer gas. Hence, images obtained with krypton-81m scanning are both convection- and diffusion-dependent. Infants tend to breathe near the closing volume of the lung, and regional differences in the distribution of the closing volume exist causing partial atelectasis of the dependent lung in spontaneously breathing and sedated infants [2, 27]. In the study by HEAF et al. [25], the investigated subjects were sedated and had lung disease. Therefore, their findings of preferential ventilation of the nondependent lung may not apply to healthy nonsedated infants. The differences in regional amplitudes between the anterior and posterior compartments can be further explained by the artificial division of the chest into two compartments. The anterior chest compartment contains the heart as a "nonlung" structure. In the supine position, gravity causes the heart to be

TABLE 1 Filling indices (FI) for the anterior and posterior lung of 10 breaths prior to and after a sigh, and for the sigh itself

\begin{tabular}{|c|c|c|c|c|c|c|}
\hline \multirow[t]{2}{*}{ Age } & \multicolumn{2}{|c|}{ Pre-sigh } & \multicolumn{2}{|c|}{ Sigh } & \multicolumn{2}{|c|}{ Post-sigh } \\
\hline & Anterior & Posterior & Anterior & Posterior & Anterior & Posterior \\
\hline 2 weeks & $1.063(0.010)$ & $0.966(0.005)$ & $0.953(0.044)$ & $1.034(0.020)$ & $1.087(0.014)$ & $0.957(0.007)$ \\
\hline 3 months & $1.092(0.008)$ & $0.931(0.007)$ & $0.927(0.045)$ & $1.072(0.036)$ & $1.098(0.010)$ & $0.930(0.007)$ \\
\hline 6 months & $1.153(0.031)$ & $0.912(0.020)$ & $0.960(0.051)$ & $1.053(0.046)$ & $1.174(0.031)$ & $0.901(0.015)$ \\
\hline
\end{tabular}

Data are presented as mean $(95 \% \mathrm{Cl})$. The $\mathrm{FI}$ was significantly different between lung anterior and posterior regions, and during the sigh ( $\mathrm{p}<0.001)$. 
suspended from the sternum, occupying a larger space in the anterior compartment, whereas in the prone position, the heart is resting on the sternum, allowing more anterior lung expansion. Our EIT data set will provide a valuable comparison for future studies in subjects with lung disease or during mechanical ventilation, where alveolar recruitment and derecruitment during tidal breathing can occur [26, 28-30].

\section{Time-course analysis}

40 yrs ago, regional filling characteristics of the lung were first described in healthy adults using radiolabelled isotopes [10]. Theoretically, if lung regions fill homogeneously, the degree of expansion will be consistent throughout the entire lung. It was found that, in an upright position, the change in lung volume (expressed as a percentage of total lung capacity) observed during an inspiratory vital capacity (VC) manoeuvre was greater in the lower regions than in the upper lung regions, and that the right lung filled earlier during a deep inhalation. Asynchrony in regional lung filling was found by KOLER et al. [31] using differential bronchospirometers in animal experiments, confirming that lung regions may not fill synchronously. EIT allows the measurement of the asynchronous filling and emptying of different lung regions [12]. In the present study, we demonstrated that the dependent lung filled before the nondependent lung, which is consistent with the lung model proposed by MiLIC-EMILI et al. [10] that models the lung as a suspended spring in gravity, with the dependent parts of the spring showing easier expansion. MiLIC-EMILI et al. [10] showed that, during the initial phase of a VC manoeuvre, the rate of volume change was greatest in the nondependent lung, whereas during the end-phase of the inspiration the greatest volume change was found in the dependent lung. In our cohort of infants, we found a similar regional rate of impedance change for all analysed sighs. During tidal breathing, the opposite behaviour was found, with the greatest rate of impedance change during the initial phase of the inspiration in the posterior lung and the greatest rate of impedance change during the end-phase of the inspiration in the anterior lung. To explain these differences, one must consider the mechanism of lung expansion during a VC manoeuvre and the contribution of the diaphragm during tidal breathing. In infants, most of the tidal volume during spontaneous breathing is generated by diaphragm excursion [32] and, to a lesser degree, by the chest wall [33]. During tidal breathing, the posterior part of the diaphragm shows the greatest shortening of muscle fibres and, hence, the rate of change in lung volume in these regions will be greater than in the anterior parts of the lung [34]. During a sigh, the contribution of the chest wall will proportionally increase to generate the large inspiratory effort [35]. The posterior part of the chest will experience proportionally less excursion than the anterior, as the posterior chest wall is splinted by the surface the infant is lying on in supine position [36]. The greater change of the anterior lung volume at the beginning of a sigh is caused by chest wall geometry and compliance in the supine position.

\section{Limitations}

The observed changes in impedance, both during tidal breathing and as an effect of growth, may not be only contributed by change in air volume, but also by change in blood volume and lung tissue characteristics. The separation of several tissue impedance characteristics can only be obtained with a multifrequency EIT equipment, which currently is not yet available for clinical use. In theory, with a multifrequency EIT, the tissue to air ratio of the lung could be separated and lung growth documented.

\section{Conclusion}

Regional ventilation distribution measurements using EIT at 2 weeks, and 3 and 6 months of age showed that the dependent lung is preferentially ventilated and fills ahead of the rest of the lung, a breathing pattern that is similar to adults.

\section{SUPPORT STATEMENT}

The study was supported by the Golden Casket and Preston James Funds.

\section{STATEMENT OF INTEREST}

None declared.

\section{REFERENCES}

1 Baldwin DN, Suki B, Pillow JJ, et al. Effect of sighs on breathing memory and dynamics in healthy infants. J Appl Physiol 2004; 97: 1830-1839.

2 Reid LM. Lung growth in health and disease. Br J Dis Chest 1984; 78: 113-134.

3 Pillow JJ, Frerichs I, Stocks J. Lung function tests in neonates and infants with chronic lung disease: global and regional ventilation inhomogeneity. Pediatr Pulmonol 2006; 41: 105-121.

4 Schibler A, Frey U. Role of lung function testing in the management of mechanically ventilated infants. Arch Dis Child 2002; 87: F7-F10.

5 Schibler A, Schneider M, Frey U, et al. Moment ratio analysis of multiple breath nitrogen washout in infants with lung disease. Eur Respir J 2000; 15: 1094-1101.

6 Pillow JJ, Ljungberg H, Hulskamp G, et al. Functional residual capacity measurements in healthy infants: ultrasonic flow meter versus a mass spectrometer. Eur Respir J 2004; 23: 763-768.

7 Schibler A, Frey U. Role of lung function testing in the management of mechanically ventilated infants. Arch Dis Child Fetal Neonatal Ed 2002; 87: F7-F10.

8 Schibler A, Hall GL, Businger F, et al. Measurement of lung volume and ventilation distribution with an ultrasonic flow meter in healthy infants. Eur Respir J 2002; 20: 912-918.

9 Gattinoni L, Caironi P, Valenza F, et al. The role of CT-scan studies for the diagnosis and therapy of acute respiratory distress syndrome. Clin Chest Med 2006; 27: 559-570.

10 Milic-Emili J, Henderson JA, Dolovich MB, et al. Regional distribution of inspired gas in the lung. J Appl Physiol 1966; 21: 749-759.

11 Bodenstein M, David M, Markstaller K. Principles of electrical impedance tomography and its clinical application. Crit Care Med 2009; 37: 713-724.

12 Dunlop S, Hough J, Riedel $\mathrm{T}$, et al. Electrical impedance tomography in extremely prematurely born infants and during high frequency oscillatory ventilation analysed in the frequency domain. Physiol Meas 2006; 27: 1151-1165.

13 Grant CA, Fraser JF, Dunster KR, et al. The assessment of regional lung mechanics with electrical impedance tomography: a pilot study during recruitment manoeuvres. Intensive Care Med 2009; 35: 166-170.

14 Thoracic Society of Australia and New Zealand, Australasian Sleep Association. Accreditation of Sleep Disorders Services, Including Standards for Paediatric Laboratories. Blacktown, Australasian Sleep Association, 2000. 
15 Frerichs I, Schiffmann H, Oehler R, et al. Distribution of lung ventilation in spontaneously breathing neonates lying in different body positions. Intensive Care Med 2003; 29: 787-794.

16 Hahn G, Frerichs I, Kleyer M, et al. Local mechanics of the lung tissue determined by functional EIT. Physiol Meas 1996; 17: Suppl. 4A, A159-A166.

17 Frerichs I, Pulletz S, Elke G, et al. Assessment of changes in distribution of lung perfusion by electrical impedance tomography. Respiration 2009; 77: 282-291.

18 Schibler A, Yuill M, Parsley C, et al. Regional ventilation distribution in non-sedated spontaneously breathing newborns and adults is not different. Pediatr Pulmonol 2009; 44: 851-858.

19 Pulletz S, van Genderingen HR, Schmitz G, et al. Comparison of different methods to define regions of interest for evaluation of regional lung ventilation by EIT. Physiol Meas 2006; 27: S115-S127.

20 Frerichs I, Dargaville PA, van Genderingen H, et al. Lung volume recruitment after surfactant administration modifies spatial distribution of ventilation. Am J Resp Crit Care Med 2006; 174: 772-779.

21 Riedel T, Richards T, Schibler A. The value of electrical impedance tomography in assessing the effect of body position and positive airway pressures on regional lung ventilation in spontaneously breathing subjects. Intensive Care Med 2005; 31: 1522-1528.

22 Prisk GK, Hammer J, Newth CJ. Techniques for measurement of thoracoabdominal asynchrony. Pediatr Pulmonol 2002; 34: 462-472.

23 Grant CA, Fraser JF, Dunster KR, et al. The assessment of regional lung mechanics with electrical impedance tomography: a pilot study during recruitment manoeuvres. Intensive Care Med 2009; 35: 166-170.

24 Brown BH, Primhak RA, Smallwood RH, et al. Neonatal lungs: maturational changes in lung resistivity spectra. Med Biol Eng Comput 2002; 40: 506-511.
25 Heaf DP, Helms P, Gordon I, et al. Postural effects on gas exchange in infants. New Engl J Med 1983; 308: 1505-1508.

26 Steinberg J, Schiller HJ, Halter JM, et al. Tidal volume increases do not affect alveolar mechanics in normal lung but cause alveolar overdistension and exacerbate alveolar instability after surfactant deactivation. Crit Care Med 2002; 30: 2675-2683.

27 Burri PH. Postnatal Development and Growth. 2nd Edn. Philadelphia, Scientific Foundations, 1997.

28 Carney DE, Bredenberg CE, Schiller HJ, et al. The mechanism of lung volume change during mechanical ventilation. Am J Resp Crit Care Med 1999; 160: 1697-1702.

29 McCann UG II, Schiller HJ, Carney DE, et al. Visual validation of the mechanical stabilizing effects of positive end-expiratory pressure at the alveolar level. J Surg Res 2001; 99: 335-342.

30 Schiller HJ, McCann UG 2nd, Carney DE, et al. Altered alveolar mechanics in the acutely injured lung. Crit Care Med 2001; 29: 1049-1055.

31 Koler JJ, Young AC, Martin CJ. Relative volume changes between lobes of the lung. J Appl Physiol 1959; 14: 345-347.

32 Agostoni E. Volume-pressure relationships of the thorax and lung in the newborn. J Appl Physiol 1959; 14: 909-913.

33 Openshaw P, Edwards S, Helms P. Changes in rib cage geometry during childhood. Thorax 1984; 39: 624-627.

34 Laing IA, Teele RL, Stark AR. Diaphragmatic movement in newborn infants. J Pediatr 1988; 112: 638-643.

35 Gaulthier C. Respiratory muscle function in infants. Eur Respir J 1995; 8: 150-153.

36 Gauthier AP, Verbanck S, Estenne M, et al. Three-dimensional reconstruction of the in vivo human diaphragm shape at different lung volumes. J Appl Physiol 1994; 76: 495-506. 